\title{
Accounting for occurrences: An explanation for some novel tendencies in causal judgment from contingency information
}

\author{
Peter A. White \\ Cardiff University, Cardiff, Wales
}

\begin{abstract}
Contingency information is information about empirical associations between possible causes and outcomes. In the present research, it is shown that, under some circumstances, there is a tendency for negative contingencies to lead to positive causal judgments and for positive contingencies to lead to negative causal judgments. If there is a high proportion of instances in which a candidate cause (CC) being judged is present, these tendencies are predicted by weighted averaging models of causal judgment. If the proportion of such instances is low, the predictions of weighted averaging models break down. It is argued that one of the main aims of causal judgment is to account for occurrences of the outcome. Thus, a CC is not given a high causal judgment if there are few or no occurrences of it, regardless of the objective contingency. This argument predicts that, if there is a low proportion of instances in which a CC is present, causal judgments are determined mainly by the number of Cell A instances (i.e., CC present, outcome occurs), and that this explains why weighted averaging models fail to predict judgmental tendencies under these circumstances. Experimental results support this argument.
\end{abstract}

Causal relations imply regularities of association between causes and their outcomes. A cause must be present if it produces its outcome, but a candidate cause (CC) need not be present if the outcome does not occur or if the outcome is caused by something else. Therefore, if an outcome occurs and its cause is uncertain, regularity of association could be used as an empirical indicator of the likely cause. A cause must have temporal priority over its outcome, meaning that it must be present either before or when the outcome occurs, so the kind of empirical association that could be used for causal inference is contingency, a time-ordered kind of regularity. When a single $\mathrm{CC}$ of an outcome is under consideration, there are four kinds of contingency information: $\mathrm{CC}$ present, outcome occurs (called Cell A); CC present, outcome does not occur (Cell B); CC absent, outcome occurs (Cell C); and CC absent, outcome does not occur (Cell D). An objective measure of contingency is provided by the $\Delta P$ rule, according to which contingency is the probability of the outcome's occurring if the $\mathrm{CC}$ is present minus the probability of the outcome's occurring if the CC is absent (McKenzie, 1994). Values of $\Delta P$ vary from -1 (perfect negative contingency) to +1 (perfect positive contingency).

People could make causal judgments by assessing objective contingencies, in which case, their causal judgments would be well predicted by the $\Delta P$ rule. Some studies have suggested that this might be so (e.g., Wasserman, Elek, Chatlosh, \& Baker, 1993). However, a recent review and meta-analysis has confirmed that causal judgments tend to diverge from values of $\Delta P$ in predictable ways (Perales \& Shanks, 2007). The conclusion from that review was that observed tendencies in causal judgment are best accounted for by weighted averaging models of causal judgment (Busemeyer, 1991; Catena, Maldonado, \& Cándido, 1998; White, 2003b, 2004).

An example of a weighted averaging model is the proportion of confirming instances $(p C I)$ model (White, 2002, $2003 \mathrm{~b}, 2004)$. The underlying principle of this model is that people treat the different kinds of contingency information as evidence about a causal hypothesis. Evidence can be confirmatory, disconfirmatory, or irrelevant. Judgment of the $\mathrm{CC}$ therefore depends on the relative amounts of confirmatory and disconfirmatory evidence available. The total of the disconfirmatory evidence is subtracted from the total of the confirmatory evidence, and the result is divided by the total amount of relevant evidence available. Cell A and Cell D information is normatively confirmatory, because instances of those kinds tend to increase the objective contingency; Cell B and Cell C information is normatively disconfirmatory, because instances of those kinds tend to decrease the objective contingency. Therefore, the simplest version of the model involves adding the frequencies in Cells A and D, subtracting those in Cells B and C, and dividing the result by the total of the frequencies in the four cells. As with $\Delta P$, values of the $p C I$ rule range from -1 to +1 , with +1 being the highest possible causal judgment.

Values of $p C I$ are isomorphic with values of $\Delta P$ if there are equal numbers of $\mathrm{CC}$-present and -absent instances. This feature of the model accounts for evidence that causal judgments are often closely attuned to $\Delta P$ values (Wasser-

P. A.White, whitepa@cardiff.ac.uk 


$$
J_{\mathrm{C} 1}=\frac{(.15 n \mathrm{C} 1 \mathrm{C} 2+)-(.075 n \mathrm{C} 1 \mathrm{C} 2-)+(.4 n \mathrm{C} 1+)-(.15 n \mathrm{C} 1-)-(.15 n \mathrm{C} 2+)-(.075 n 0+)}{(.15 n \mathrm{C} 1 \mathrm{C} 2+)+(.075 n \mathrm{C} 1 \mathrm{C} 2-)+(.4 n \mathrm{C} 1+)+(.15 n \mathrm{C} 1-)+(.15 n \mathrm{C} 2+)+(.075 n 0+)}
$$

man et al., 1993; White, 2003b). If numbers of CC-present and -absent instances are not equal, values of $p \mathrm{CI}$ and $\Delta P$ tend to diverge. This tendency has motivated the design of several tests of the $p$ CI rule. It has been shown that, if $p \mathrm{CI}$ values are manipulated and $\Delta P$ values are held constant, causal judgments tend to vary in accordance with the changes in $p C I$ values, but that if $\Delta P$ values are manipulated and $p C I$ values are held constant, little or no difference in causal judgment occurs (White, 2002, 2003b, 2004).

The $p$ CI rule is an oversimplification, because it assigns equal weights to all four cells. Several studies have shown that the four cells do not carry equal weight in causal judgment. The usual finding is that $\mathrm{A}>\mathrm{B}>\mathrm{C}>\mathrm{D}$ (Anderson \& Sheu, 1995; Kao \& Wasserman, 1993; Levin, Wasserman, \& Kao, 1993; Mandel \& Lehman, 1998; Wasserman, Dorner, \& Kao, 1990; Wasserman, Kao, Van Hamme, Katagiri, \& Young, 1996; White, 2003a). White (2004) set weights in accordance with the findings of previous research and found that a model with those weights, termed $w p C I$ (weighted proportion of confirming instances), was a better predictor of causal judgment than was the original $p$ CI rule (see also Perales \& Shanks, 2007).

However, weighted averaging models predict some judgmental tendencies that are most unlikely to be observed. Consider the following set of cell frequencies: Cell $\mathrm{A}=0$; Cell $\mathrm{B}=10$; Cell $\mathrm{C}=10$; Cell $\mathrm{D}=100$. For this set, $\Delta P=-.09$, suggesting a weak negative contingency. However, $p \mathrm{CI}=+.67$, suggesting a moderately strong positive judgment. Using the weights used by White (2004), $w p C I=+.38$, a lower, but still positive, value. Increase the number of Cell D instances in the set to, say, 100,000, and weighted averaging models would produce values close to their theoretical ceilings. Yet it is very unlikely that a positive causal judgment would be observed for this set of data, only because there are no Cell A instances. How could a candidate be judged to cause an outcome for cases in which the outcome never occurs in the presence of the candidate? Addressing this issue provides at least a partial explanation for the observed differences in cell weights and also helps to predict when weighted averaging models will and will not predict tendencies in causal judgment.

In White (2008), I put forward the accounting for occurrences (AFO) model, in which it is proposed that there are two main aims of causal judgment and that contingency information is used in the service of both. The two aims are estimating the strengths of CCs and judging the extent to which a given $\mathrm{CC}$ accounts for occurrences of an outcome. Estimating strength involves giving most weight to Cell A and Cell B information: A stronger cause tends to produce its outcome more often than a weaker cause does, leading to a higher proportion of Cell A to Cell B instances. This is not a normative procedure; it is argued in White (2008) that this is how it is usually done, but not that it is how it is invariably or properly done. Accounting for occurrences involves assessing the extent to which the $\mathrm{CC}$ being judged is present for cases in which the outcome occurs. A CC can account for outcomes that occur for cases in which it is present but not for outcomes for which it is not. This implies that most weight should be given to Cells A and C, with Cell A being treated as confirmatory and Cell $\mathrm{C}$ being treated as disconfirmatory.

In White (2008), these principles were formalized as a weighted averaging model of judgment in which the weight given to each kind of contingency information reflected its importance for the two aims of judgment, in accordance with the foregoing summary. That model was concerned with the case in which two explicit CCs, $\mathrm{C} 1$ and $\mathrm{C} 2$, are under consideration. In that case, there are eight kinds of contingency information, identified as $\mathrm{C} 1 \mathrm{C} 2+$ and $\mathrm{C} 1 \mathrm{C} 2-$ (occurrences and nonoccurrences; $\mathrm{C} 1$ and $\mathrm{C} 2$ both present), $\mathrm{C} 1+$ and $\mathrm{C} 1-$ (occurrences and nonoccurrences; $\mathrm{C} 1$ present, $\mathrm{C} 2$ absent), $\mathrm{C} 2+$ and $\mathrm{C} 2-$ (occurrences and nonoccurrences; $\mathrm{C} 2$ present, $\mathrm{C} 1$ absent), and $0+$ and $0-$ (occurrences and nonoccurrences; both CCs absent). The model is shown in Equation 1 (see above).

$J_{\mathrm{C} 1}$ stands for judgment of candidate $\mathrm{C} 1$, and $n$ stands for the number of instances of a given kind. For judgment of candidate $\mathrm{C} 2$, substitute $\mathrm{C} 2+$ for $\mathrm{C} 1+, \mathrm{C} 2-$ for $\mathrm{C} 1-$, and $\mathrm{C} 1+$ for $\mathrm{C} 2+$. Weights are normalized to sum to 1 , and the equation generates values in the range -1 (prevent) to +1 (cause). For judgments of $\mathrm{C} 1, \mathrm{C} 1 \mathrm{C} 2+$ and $\mathrm{C} 1+$ have positive valence; the other four kinds of information in the model have negative valence. $\mathrm{C} 2-$ and $0-$ are not represented, because, under the AFO hypothesis, they carry negligible weight.

A first approximation to a model for the case in which just one CC is under consideration can be extracted from Equation $1 . \mathrm{Cl}+$ (where $\mathrm{C} 1$ is being judged) is equivalent to Cell A, C1 - to Cell B, 0 + to Cell C, and 0- to Cell D. This yields the model shown in Equation 2:

$$
J_{\mathrm{A}}=\frac{(.4 n \mathrm{~A})-(.15 n \mathrm{~B})-(.075 n \mathrm{C})}{(.4 n \mathrm{~A})+(.15 n \mathrm{~B})+(.075 n \mathrm{C})} .
$$

The model implies a rank ordering of cell weights of $\mathrm{A}>\mathrm{B}>\mathrm{C}>\mathrm{D}$, which is consistent with the evidence discussed above. However, the model assigns zero weight to Cell D information. In the two-CC case modeled by Equation 1, this weighting appears to be justified, because 0 - information carries negligible weight in causal judgment (White, 2008). For cases in which one CC is under consideration, however, it has been found that Cell D carries nonzero weight, even though it carries less weight than do the other three cells (Anderson \& Sheu, 1995; Kao \& Wasserman, 1993; Levin et al., 1993; Mandel \& Lehman, 1998; Wasserman et al., 1990; Wasserman et al., 1996; White, 2003a). This issue is addressed in the General Discussion section, in the light of the results reported herein. 


\section{The Problem of Low Numbers of CC-Present Instances}

The AFO principle implies that causal judgment will never be high if there are few or no Cell A instances, because, in that case, the $\mathrm{CC}$ clearly does not account for many occurrences of the outcome. Other things being equal, the more Cell A instances there are, the higher the causal judgment should be. This is a key claim with regard to the $p \mathrm{CI}$ and $w p C I$ rules. The predictions of those models diverge from those generated by the $\Delta P$ rule for cases in which numbers of CC-present and -absent instances are not equal. There can be two kinds of such inequality: more CC-present than -absent instances and more CC-absent than -present instances. Let the proportion of CC-present instances in the set of instances under consideration be designated by $p(\mathrm{C})$. Then the predictions generated by $p \mathrm{CI}$ and $w p \mathrm{CI}$, on the one hand, and the $\Delta P$ rule, on the other, diverge increasingly as $p(\mathrm{C})$ increases above .5 and as it decreases below .5 . Thus, extreme values of $p(\mathrm{C})$ below .5 are important to the aim of distinguishing among the $p \mathrm{CI}, w p \mathrm{CI}$, and $\Delta P$ rules. However, at such values, small numbers of Cell A instances tend to occur. The AFO model therefore predicts that $w p \mathrm{CI}$ in particular is a good predictor of causal judgments at high values of $p(\mathrm{C})$, but that its predictions tend to break down at low values of $p(C)$ because they are not sensitive to the problems posed by low numbers of Cell A instances. No matter how high the values of $p \mathrm{CI}$ and $w p \mathrm{CI}$ may be, the AFO model predicts that causal judgments will never be high if there are few or no Cell A instances. One aim of the present research is therefore to test the predictions of the $p \mathrm{CI}$ and $w p \mathrm{CI}$ rules for sets with low and high values of $p(C)$ in order to ascertain whether the failures of these rules that are predicted by the AFO model occur. The implications of this for the model shown in Equation 2 are addressed in the General Discussion section.

\section{Counternormative Tendencies in Causal Judgment}

The manipulation of $p(\mathrm{C})$ also permits a striking test of the claim, shared by the AFO model and the $p \mathrm{CI}$ and $w p \mathrm{CI}$ rules, that differences in causal judgment contrary to differences in the objective contingency can occur at extreme values of $p(\mathrm{C})$. Specifically, a set of data with a negative contingency can receive a causal judgment higher than that of a set of data with a positive contingency. Observation of such a tendency would be an important disconfirmation of the view that causal judgment tends to conform to normative standards of causal inference.

Consider the power PC theory (Cheng, 1997). This theory is grounded in a normative analysis of the conditions under which causal inferences can be made from contingency information. Take the case in which a given outcome can be produced by more than one cause. To identify whether a CC is the cause of the outcome, its contribution to the outcome must be distinguished from that of the set (S) of the other possible causes. The power of $\mathrm{S}$ is estimated by the baserate probability, the probability with which the outcome occurs if the $\mathrm{CC}$ is absent. The question is whether the $\mathrm{CC}$ makes a difference to the incidence of the outcome over and above the base-rate probability. Given certain assumptions, such as that the probabilities of the $\mathrm{CC}$ and $\mathrm{S}$ are distributed independently of each other and that $\Delta P \leq 0$, the $\mathrm{CC}$ could be a generative cause. In that case, its generative power is estimated by dividing $\Delta P_{\mathrm{CC}}$ by 1 minus the base-rate probability. If $\Delta P \geq 0$, the $\mathrm{CC}$ could be a preventive cause, and its preventive power is estimated by dividing $\Delta P_{\mathrm{CC}}$ by the base-rate probability. These estimates are termed "probabilistic power" and indicated by $p .{ }^{1}$ The argument to this inferential procedure is presented in full in Cheng. The relevant point for the present purposes is that it is claimed to be a valid inferential procedure, "a solution to the problem of causal induction first posed by Hume" (Cheng, 1997, p. 398). Furthermore, "it $i$ s the solution adopted biologically by humans" (Cheng, 1997, p. 398, italics in original).

The power PC theory does not predict that human causal judgment is always optimal by the standards set by the theory. The inferential procedure in the power PC theory is inductive, and inductive inferences can never be assumed to guarantee objectively correct conclusions. Given a limited sample of evidence, the inferential procedure may fail to identify a cause if the cause is weak or if the base-rate probability is high. Causal inferences are valid only if the requisite assumptions are met, so faulty causal inferences can be made if a reasoner believes that the assumptions hold when they do not. Furthermore, human judgment can be led astray by factors extrinsic to the process of causal judgment, such as limitations on processing capacity, methodological factors in experiments, and influence from other mental processes and states of belief. So the power PC theory does not predict that human causal judgment will always be optimal by the standards of the model.

Nevertheless, it is possible to identify certain tendencies in causal inference that should not occur, according not only to the power PC theory but also to any theory or model under which human causal inference is postulated to be normatively appropriate. It would be strongly disconfirmatory for the normative hypothesis if human judges consistently identified one $\mathrm{CC}$ as generative if it had an objectively negative contingency with an outcome, identified one as preventive if it had an objectively positive contingency with an outcome, and the judged causal status of the two CCs was in the opposite direction from that of the difference in their respective contingencies. It is hard to imagine any normative model under which something that makes the outcome less likely to occur could be validly identified as a generative $\mathrm{CC}$ of it, or something that makes the outcome more likely to occur could be validly identified as a preventer for it. In this research, I report evidence for exactly these tendencies, and I show that the AFO model can predict whether they will or will not occur.

\section{EXPERIMENT 1}

\section{Method}

Participants. Forty-four first-year undergraduate students of psychology at Cardiff University participated in return for course credit. None had been taught any psychology of relevance to the topic of the experiment. 
Table 1

Cell Frequencies, Values of Independent Variables, $\Delta P$, Proportion

of Confirming Instances ( $p C I)$, Weighted Proportion of Confirming Instances (wpCI), Probabilistic Power ( $p)$, Support, and Observed Causal Judgments, Experiment 1

\begin{tabular}{|c|c|c|c|c|c|c|c|c|}
\hline$\frac{\text { Cell }}{\mathrm{A}, \mathrm{B}, \mathrm{C}, \mathrm{D}}$ & $p(\mathrm{C})$ & $p(\mathrm{E})$ & $\Delta P$ & $p \mathrm{CI}$ & $w p \mathrm{CI}$ & $p$ & Support & $\begin{array}{c}\text { Mean } \\
\text { Judgment }\end{array}$ \\
\hline \multicolumn{9}{|c|}{ Group 1} \\
\hline $1,4,8,24$ & .14 & .24 & -.05 & +.35 & .00 & -.2 & -0.56 & -10.61 \\
\hline $4,1,24,8$ & .14 & .76 & +.05 & -.35 & -.46 & +.2 & -0.56 & -2.73 \\
\hline $8,24,1,4$ & .86 & .24 & +.05 & -.35 & -.33 & +.2 & -1.10 & -27.98 \\
\hline $24,8,4,1$ & .86 & .76 & -.05 & +.35 & +.47 & -.2 & -1.10 & 52.32 \\
\hline \multicolumn{9}{|c|}{ Group 2} \\
\hline $0,2,8,24$ & .06 & .24 & -.25 & +.41 & +.04 & -1.0 & +0.68 & -19.41 \\
\hline $2,0,24,8$ & .06 & .76 & +.25 & -.41 & -.58 & +1.0 & +0.68 & -14.86 \\
\hline $8,24,0,2$ & .94 & .24 & +.25 & -.41 & -.34 & +1.0 & -0.96 & -27.39 \\
\hline $24,8,2,0$ & .94 & .76 & -.25 & +.41 & +.53 & -1.0 & -0.96 & 42.07 \\
\hline
\end{tabular}

Note $-p(\mathrm{C})=$ the proportion of instances in which the cause is present; $p(\mathrm{E})=$ the proportion of instances in which the effect occurs; and $p(\mathrm{C})$ and $p(\mathrm{E})$ are the independent variables in the two designs. Judgment $=$ obtained mean causal judgments.

Stimulus materials. This experiment used the instance list method (White, 2002, 2004), in which participants are presented with a cover story followed by a series of sets of evidence, hereafter called data sets, each on a separate page. Within data sets, instances are presented individually but organized in a tabular presentation with each row describing the properties of one instance. An example data set is shown in the Appendix. This is the first data set in Group 1, as shown in Table 1.

An initial instruction sheet introduced the cover story: A kind of fish, the polychrome parrotfish, was usually uniformly red, but some individuals had bright green spots. Marine biologists were testing the hypothesis that a trace mineral (manganese sulfate; here, MS) sometimes found in seawater might either cause or prevent these spots if it was present when the eggs were laid. The scientists set up a lot of aquaria, each containing one parrotfish egg, and they controlled whether the water in each aquarium contained MS. They then looked to see whether the fish developed green spots.

Participants were then told that each subsequent page presented the results of a test for different species of polychrome parrotfish. At the top of the page, a number identified the species. Under this were three columns of information. The left column only numbered the instances (aquariums). The middle column recorded whether MS was present in each aquarium. The right column recorded whether the fish developed green spots. At the bottom of each page, participants were asked, "How does MS affect the occurrence of green spots in this species of parrotfish?" They were then instructed to answer this question by writing a number from -100 to +100 beside it, where -100 meant that MS causes a great decrease in the occurrence of green spots in the species being judged, +100 meant that it causes a great increase in the occurrence of green spots, and 0 meant that it has no effect. Participants were informed that they could write any number between -100 and +100 .

The subsequent pages presented the individual data sets in the format described above and exemplified in the Appendix.

Design. Data sets were constructed with high and low values of $p(C)$. The required separation of $p C I$ and $\Delta P$ values could then be accomplished by manipulating the proportion of instances in which the effect occurred, $p(\mathrm{E})$. Two different groups of such manipulations were constructed for this experiment, each comprising four data sets. Table 1 depicts the effects of these manipulations on values of $\Delta P$ and $p C I$. The first four data sets in Table 1 show Group 1, in which $\Delta P$ takes values of +.05 in two data sets and -.05 in the other two. The other four data sets in Table 1 constitute Group 2, in which $\Delta P$ takes values of +.25 in two data sets and -.25 in the other two. Values of $w p C I$, also shown in Table 1, were derived using the same weights as in White (2004). Cells A and D had positive valence with weights of .72 and .23 , respectively. Cells B and C had negative valence with weights of .53 and .51 , respectively.

Table 1 shows that the data sets in which $\Delta P$ takes a positive value are those in which $p \mathrm{CI}$ and $w p \mathrm{CI}$ take negative values, and vice versa (with one exception in which $w p C I=0$ ). It is predicted that, for the data sets with high $p(C)$ (the third and fourth data sets in each group, as shown in Table 1), causal judgments should tend to vary in accordance with $w p$ CI values; that is, they should tend to be both higher and above zero if $w p \mathrm{CI}$ is high and lower and below zero if $w p \mathrm{CI}$ is low, contrary to the difference in the objective contingencies. If $p(\mathrm{C})$ is low, however, Table 1 shows that there are few Cell A instances. Specifically, there are fewer Cell A instances in the data sets with high values of $p C I$ and $w p C I$ (the first data set in each group) than in those with low values (the second data set in each group). Because of this, the AFO model predicts that causal judgments should not show the pattern predicted by $p \mathrm{CI}$ and $w p \mathrm{CI}$ for these data sets. It predicts instead that causal judgments should tend to be low and to be positively correlated with Cell A frequency.

Table 1 shows values of $p$. These are invariably positive if $\Delta P$ is positive and negative if $\Delta P$ is negative. This shows that the predictions of the power PC theory are also contrary to those of the $p \mathrm{CI}$ and $w p \mathrm{CI}$ rules, as would be expected of a normative model in this case.

Each data set in Group 1 had 37 instances. Each data set in Group 2 had 34 instances.

Procedure. Participants took part individually or in groups of 2 or 3. Participants in groups were positioned so that none could see what the others were doing. The materials for this experiment were included with those for two other experiments on unrelated topics. Participants were supervised by an experimenter who introduced the experiments, handed out informed consent forms, and invited participants to ask questions if anything in the instructions was not clear. There were no questions concerning the present experiment. At the end, participants were thanked and were given course credit and a debriefing sheet that explained the general aims of the research but not the specific hypothesis.

\section{Results}

Individual data sets are identified by their cell frequencies in the order $\{A, B, C, D\}$, as shown in Table 1. Mean causal judgments are shown in Table 1 . When $p(\mathrm{C})$ was high (.86 in Group 1, .94 in Group 2), the means showed close agreement with $p$ CI and $w p C I$ values. The tenden- 
cies were in each case confirmed by a chi-square test on numbers of participants giving positive and negative judgments. For Group 1, data set $\{8,24,1,4\}$, there was a significant tendency for negative judgments to be given $\left[\chi^{2}(1)=11.00, p<.01\right]$. For Group 1 , data set $\{24,8,4,1\}$, there was a significant tendency for positive judgments to be given $\left[\chi^{2}(1)=38.05, p<.01\right]$. For Group 2, data set $\{8,24,0,2\}$, there was a significant tendency for negative judgments to be given $\left[\chi^{2}(1)=\right.$ $8.40, p<.01]$. For Group 2 , data set $\{24,8,2,0\}$, there was a significant tendency for positive judgments to be given $\left[\chi^{2}(1)=26.56, p<.01\right]$. In all four cases, these tendencies were contrary to the direction of the objective contingency.

When $p(C)$ was low (.14 in Set 1 and .06 in Set 2), mean judgments were all below zero and judgmental tendencies did not conform to the predictions of $p \mathrm{CI}, w p \mathrm{CI}, \Delta P$, or the power PC theory. For Group 1, data set $\{1,4,8,24\}$, there was a significant tendency for negative judgments to be given $\left[\chi^{2}(1)=5.76, p<.05\right]$. For Group 1 , data set $\{4,1,24,8\}$, there was no significant result $\left[\chi^{2}(1)=\right.$ $0.44]$. For Group 2, data set $\{0,2,8,24\}$, there was a significant tendency for negative judgments to be given $\left[\chi^{2}(1)=7.75, p<.01\right]$. For Group 2, data set $\{2,0,24,8\}$, there was no significant result $\left[\chi^{2}(1)=0.14\right]$.

\section{Discussion}

For the four data sets with high values of $p(\mathrm{C})$, the results were in close agreement with $p \mathrm{CI}$ and $w p \mathrm{CI}$ values and contrary to $\Delta P$ and $p$ values. There was a significant tendency to give negative judgments to the two data sets with positive values of $\Delta P$ and to give positive judgments to the two data sets with negative values of $\Delta P$. These results disconfirm the predictions of the power PC theory that sets of evidence with positive values of $\Delta P$ should be given positive causal judgments and sets with negative values of $\Delta P$ should be given negative judgments. Contrary to any plausible normative analysis, candidates are sometimes judged to be causes in cases where they are negatively associated with the outcome, and sometimes judged to be preventers in cases where they are positively associated with the outcome. In this study, the predictions of $p \mathrm{CI}$ and $w p \mathrm{CI}$ are very similar and the results do not clearly favor one over the other.

For the four data sets with low values of $p(\mathrm{C})$, the results did not fit the predictions of $p$ CI or $w p C I$. All four means were below zero, and this tendency was statistically significant for the two data sets with positive values of $p \mathrm{CI}$. These were the two data sets with the lowest Cell A frequencies. These results were therefore consistent with the argument that a main aim of causal judgment is to account for occurrences of the outcome. Causal judgments would not be high for the two data sets in question, despite the predominance of confirmatory evidence in them, because there were too few occurrences of the outcome in the presence of the CC. In fact, mean causal judgments for the four data sets with low values of $p(\mathrm{C})$ were highly correlated with Cell A frequencies $(r=+.88)$, indicating a tendency for judgments to rise as Cell A frequency increased. This is consistent with the AFO interpretation.

Experiment 2 was designed as a further test of the predicted counternormative tendency with high values of $p(\mathrm{C})$. Experiments 3 and 4 were designed as a further test of the hypothesis that the AFO model predicts the judgmental tendencies observed when $p(\mathrm{C})$ is low.

\section{EXPERIMENT 2}

\section{Method}

Experiment 2 differed from Experiment 1 in only the following details. None of the 40 participants had participated in Experiment 1. There were two independent variables. One was a manipulation of the objective contingency by altering values of $p(\mathrm{E} / \mathrm{C})$ and $p(\mathrm{E} /-\mathrm{C})$, the probability of the outcome's occurring if the $\mathrm{CC}$ is present and if it is absent, respectively. A contingency of $\Delta P=+.25$ was created by setting $p(\mathrm{E} / \mathrm{C})=.25$ and $p(\mathrm{E} /-\mathrm{C})=0$. A contingency of $\Delta P=$ -.25 was created by setting $p(\mathrm{E} / \mathrm{C})=.75$ and $p(\mathrm{E} /-\mathrm{C})=1$. The other variable was a manipulation of sample size with two values, 10 and 40 . The aim of this was to test the causal support model (Griffiths \& Tenenbaum, 2005), which predicts effects of this variable. This model is assessed in the General Discussion section. Values of the sample size variable, along with values of $\Delta P, p \mathrm{CI}, w p C \mathrm{I}, p$, and support, are shown in Table 2 . There were four data sets altogether.

\section{Results and Discussion}

Mean causal judgments are shown in Table 2. Judgments showed close agreement with $w p C I$ values and not with $\Delta P$ values. Data were analyzed with a $2(\Delta P:+.25$ vs. -.25$) \times 2$ (sample size: 10 vs. 40$)$ within-subjects ANOVA. Just one significant effect was found, a main effect of $\Delta P\left[F(1,39)=41.13, M S_{\mathrm{e}}=3,197.34, p<.001\right]$, with a higher mean at $\Delta P=-.25(26.15)$ than at $\Delta P=$ $+.25(-31.19)$. The difference in means is as predicted by $w p C I$ but opposite to the difference in $\Delta P$ and $p$ values. There was no significant effect of or interaction with sample size.

Table 2

Cell Frequencies, Values of Independent Variables, $\Delta P$, Proportion of Confirming Instances ( $p C I)$, Weighted Proportion of Confirming Instances (wpCI), Probabilistic Power ( $p$ ), Support, and Observed Causal Judgments, Experiment 2

\begin{tabular}{|c|c|c|c|c|c|c|c|}
\hline$\frac{\text { Cell }}{\mathrm{A}, \mathrm{B}, \mathrm{C}, \mathrm{D}}$ & Sample & $\Delta P$ & $p \mathrm{CI}$ & $w p \mathrm{CI}$ & $p$ & Support & $\begin{array}{c}\text { Mean } \\
\text { Judgment }\end{array}$ \\
\hline $2,6,0,2$ & 10 & +.25 & -.20 & -.28 & +.25 & -0.90 & -30.75 \\
\hline $6,2,2,0$ & 10 & -.25 & +.20 & +.39 & -.25 & -0.90 & 24.30 \\
\hline $8,24,0,8$ & 40 & +.25 & -.20 & -.28 & +.25 & -1.49 & -31.62 \\
\hline $24,8,8,0$ & 40 & -.25 & +.20 & +.39 & -.25 & +0.25 & 28.00 \\
\hline
\end{tabular}


Table 3

Cell Frequencies, Values of Independent Variables, Cell A Frequency, $\Delta P$, Proportion of Confirming Instances $(p C I)$, Weighted Proportion of Confirming Instances (wpCI), Dual-Factor Heuristic $(H)$, and Observed Causal Judgments, Experiment 3

\begin{tabular}{|c|c|c|c|c|c|c|c|c|}
\hline Cell & & & & & & & Mean & \\
\hline $\mathrm{A}, \mathrm{B}, \mathrm{C}, \mathrm{D}$ & Sample & Cell A & $\Delta P$ & $p \mathrm{CI}$ & $w p \mathrm{CI}$ & $H$ & Judgment & Predicted \\
\hline $1,0,6,2$ & 9 & 1 & +.25 & -.33 & -.44 & .38 & 9.92 & 6.72 \\
\hline $2,0,12,4$ & 18 & 2 & +.25 & -.33 & -.44 & .38 & 9.57 & 12.18 \\
\hline $3,0,18,6$ & 27 & 3 & +.25 & -.33 & -.44 & .38 & 17.21 & 15.55 \\
\hline $4,0,24,8$ & 36 & 4 & +.25 & -.33 & -.44 & .38 & 16.77 & 15.91 \\
\hline $1,0,2,6$ & 9 & 1 & +.75 & +.55 & +.35 & .58 & 22.80 & 10.40 \\
\hline $2,0,4,12$ & 18 & 2 & +.75 & +.55 & +.35 & .58 & 24.25 & 20.34 \\
\hline $3,0,6,18$ & 27 & 3 & +.75 & +.55 & +.35 & .58 & 36.05 & 29.39 \\
\hline $4,0,8,24$ & 36 & 4 & +.75 & +.55 & +.35 & .58 & 39.05 & 37.18 \\
\hline
\end{tabular}

Note- "Predicted" refers to values generated by the model for low $p(\mathrm{C})$ described in the General Discussion section.

The two data sets with $\Delta P=+.25$ were rated below zero by a significant majority of participants $\left[\chi^{2}(1)=\right.$ 7.12 , sample size $=10 ; \chi^{2}(1)=6.74$, sample size $=40$; $p<.05$ in both cases]. The two data sets with $\Delta P=-.25$ were rated above zero by a significant majority of participants $\left[\chi^{2}(1)=6.12\right.$, sample size $=10 ; \chi^{2}(1)=6.08$, sample size $=40 ; p<.05$ in both cases]. These results are therefore in agreement with those for the high $p(\mathrm{C})$ data sets in Experiment 1, showing consistently negative judgments of the positive contingencies and consistently positive judgments of the negative contingencies.

\section{EXPERIMENT 3}

The aim of Experiment 3 was to test the prediction of the AFO model that, if $p(\mathrm{C})$ is low, causal judgments would tend to increase with increases in Cell A frequency in a way not predicted by any other model. Manipulating Cell A frequency tends to affect values of other variables as well. In fact, it is impossible to manipulate Cell A frequency without changing values of any other variables, so the strategy adopted in this experiment was to devise a manipulation of Cell A frequency that involved changing the value of sample size alone and then addressing the possibility that the change in sample size might account for the results in a separate experiment (Experiment 4).

\section{Method}

Experiment 3 differed from Experiment 1 in only the following details. None of the 40 participants had participated in either of the previous experiments. There were two independent variables. One was a manipulation of the objective contingency - specifically, a manipulation of $p(\mathrm{E} /-\mathrm{C})$ while holding $p(\mathrm{E} / \mathrm{C})$ constant at 1 . A contingency of $\Delta P=+.25$ was created by setting $p(\mathrm{E} /-\mathrm{C})=.75$, and a contingency of $\Delta P=+.75$ was created by setting $p(\mathrm{E} /-\mathrm{C})=.25$. The other was a manipulation of sample size. The aim of this was to manipulate Cell A frequency with values of $1,2,3$, and 4 , which entailed commensurate proportional manipulations of frequencies in Cells $\mathrm{C}$ and $\mathrm{D}$, with Cell $\mathrm{B}=0$ in all conditions. There were eight data sets altogether.

Values of the independent variables, along with values of $\Delta P$, $p C I$, and $w p C I$, are shown in Table 3. The manipulation of sample size was designed to hold values of $\Delta P, p C I$, and $w p C I$ constant, and Table 3 shows this to be the case. Table 3 also shows values generated by the $H$ rule. This is the rule for generating causal judgments in the dual-factor heuristic proposed by Hattori and Oaksford (2007).
This rule and its predictions are addressed in the General Discussion section. For present purposes, it suffices to note that values of $H$ are not affected by the sample size manipulation. A significant effect of the sample size manipulation therefore could not be explained by any of those models whose predictions are listed in Table 3 . The AFO model predicts a significant effect of the sample size manipulation; specifically, judgment should tend to increase as sample size increases, because the number of occurrences of the outcome that can be accounted for by the CC (i.e., the number of Cell A instances) increases.

\section{Results and Discussion}

Mean causal judgments are shown in Table 3. The main result was a significant effect of the sample size manipulation consistent with the prediction based on the AFO model. Data were analyzed with a $2(\Delta P:+.25$ vs. +.75$)$ $\times 4$ (sample size: 9 vs. 18 vs. 27 vs. 36 ) within-subjects ANOVA. There was a significant effect of $\triangle P[F(1,39)=$ $\left.21.16, M S_{\mathrm{e}}=1,113.82, p<.001\right]$, with a higher mean at the higher value of $\Delta P(30.54)$ than at the lower value (13.37). There was a significant effect of sample size $\left[F(3,117)=4.42, M S_{\mathrm{e}}=688.16, p<.01\right]$. Post hoc paired comparisons with the Newman-Keuls test revealed that the means for sample sizes of $36(M=27.91)$ and $27(M=26.63)$ were significantly higher than those for sample sizes of $18(M=16.91)$ and $9(M=16.36)$.

The effect of $\Delta P$ is predicted by all the models under test here. The main result of interest is the significant effect of the sample size manipulation, which showed a trend consistent with the prediction of the AFO model. None of the other models under test predicted this result. According to the AFO model, this is an effect of the manipulation of Cell A frequency. Before this conclusion can be drawn, it is necessary to rule out the alternative possibility that it is an effect of sample size. If this were the case, it should be possible to demonstrate effects of sample size on judgment in other situations with low $p(\mathrm{C})$. Experiment 4 was designed to test this possibility.

\section{EXPERIMENT 4}

This experiment was designed as a manipulation of sample size, holding Cell A frequency constant and with an orthogonal manipulation of the objective contingency. The manipulation of sample size was effected by altering the 
Table 4

Cell Frequencies, Values of Independent Variables, Cell A Frequency, $\Delta P$, Proportion of Confirming Instances $(p C I)$, Weighted Proportion of Confirming Instances (wpCI), Dual-Factor Heuristic $(H)$, and Observed Causal Judgments, Experiment 4

\begin{tabular}{|c|c|c|c|c|c|c|c|c|}
\hline Cell & & & & & & & Mean & \\
\hline $\mathrm{A}, \mathrm{B}, \mathrm{C}, \mathrm{D}$ & Sample & Cell A & $\Delta P$ & $p \mathrm{CI}$ & $w p \mathrm{CI}$ & $H$ & Judgment & Predicted \\
\hline $1,1,6,2$ & 10 & 1 & -.25 & -.40 & -.51 & .27 & -5.67 & -0.72 \\
\hline $1,1,12,4$ & 18 & 1 & -.25 & -.44 & -.60 & .20 & -8.30 & -3.50 \\
\hline $1,1,18,6$ & 26 & 1 & -.25 & -.46 & -.64 & .16 & -7.65 & -5.50 \\
\hline $1,1,24,8$ & 34 & 1 & -.25 & -.48 & -.67 & .14 & -14.52 & -7.00 \\
\hline $1,1,2,6$ & 10 & 1 & +.25 & +.40 & +.15 & .41 & 2.00 & 3.71 \\
\hline $1,1,4,12$ & 18 & 1 & +.25 & +.44 & +.15 & .32 & -2.42 & 3.93 \\
\hline $1,1,6,18$ & 26 & 1 & +.25 & +.46 & +.15 & .27 & 1.60 & 4.09 \\
\hline $1,1,8,24$ & 34 & 1 & +.25 & +.48 & +.15 & .24 & 0.75 & 4.22 \\
\hline
\end{tabular}

number of CC-absent instances without altering $p(\mathrm{E} /-\mathrm{C})$. The AFO model predicts no effect of this manipulation because Cell A frequency is held constant. The $p \mathrm{CI}$ rule predicts an effect of the sample size manipulation, and the $w p C I$ rule predicts an interaction, with an effect of sample size at one contingency and not at the other.

\section{Method}

Experiment 4 differed from Experiment 1 in only the following details. None of the 40 participants had participated in any of the previous experiments. There were two independent variables. One was a manipulation of the objective contingency-specifically, a manipulation of $p(\mathrm{E} /-\mathrm{C})$ while holding $p(\mathrm{E} / \mathrm{C})$ constant at .5. A contingency of $\Delta P=-.25$ was created by setting $p(\mathrm{E} /-\mathrm{C})=.75$, and a contingency of $\Delta P=+.25$ was created by setting $p(\mathrm{E} /-\mathrm{C})=.25$. The other variable was a manipulation of sample size - specifically, a manipulation of the number of CC-absent instances. Total sample size took four values, 10, 18, 26, and 34. Cell A frequency was held constant at 1 . There were eight data sets altogether. Values of the independent variables, along with values of $\Delta P, p \mathrm{CI}, w p \mathrm{CI}$, and $H$ (dual-factor heuristic), are shown in Table 4.

\section{Results and Discussion}

There was just one significant result, a main effect of $\Delta P\left[F(1,39)=15.15, M S_{\mathrm{e}}=478.54, p<.001\right]$, with a higher mean at the higher value of $\Delta P(+0.48)$ than at the lower value. All the models predict this difference. The main effect of sample size was not significant $[F(3,117)=$ 1.29]. Means are presented in Table 4.

In this experiment, sample size changed while Cell A frequency remained constant, and no significant effect of the sample size manipulation was found. This result suggests that the effect of sample size found in Experiment 3 was not due to the change in total sample size, but to the change in Cell A frequency. This is consistent with the AFO model.

\section{GENERAL DISCUSSION}

The AFO model takes the understanding of causal judgment beyond the mere predictive successes of weighted averaging models. It proposes a general theoretical foundation for the unequal cell weights in causal judgment, as manifestations of the use of contingency information to meet the two main aims of causal judgment, accounting for occurrences and estimating strength. It also predicts circumstances under which the predictions of the $p \mathrm{CI}$ and $w p C I$ models break down, which exposes the limitations of averaging models in which weights are constant across different circumstances of judgment. Cell weights are statistical averages, across judges and across judgmental circumstances. Becoming able to predict deviations from those averages, such as circumstances under which judgment is dominated by Cell A frequency [low $p(\mathrm{C})$ data sets], marks a move beyond computational models toward explanatory accounts that capture the process of making a causal judgment from contingency information.

In the first two experiments, if $p(\mathrm{C})$ was high, judgments of positive contingencies were found to be negative and judgments of negative contingencies to be positive. These results were consistent with the predictions of the $p \mathrm{CI}$ and $w p C I$ models, but contrary to those of the $\Delta P$ rule and the power PC theory. In Experiment 1, if $p(\mathrm{C})$ was low, negative contingencies were judged negative by a significant majority of participants and there was no significant tendency in either direction for positive contingencies. The results for the negative contingencies were contrary to the predictions of the $p \mathrm{CI}$ and $w p \mathrm{CI}$ models. They were, however, consistent with the predictions of the AFO model. The model predicts that causal judgments tend to be low or negative whenever there are few or no Cell A instances in a given data set, as tends to be the case when $p(\mathrm{C})$ is low. In fact, mean causal judgments for the low $p(\mathrm{C})$ data sets were highly correlated with Cell A frequency, suggesting that the inferred capacity of the $\mathrm{CC}$ to account for occurrences of the outcome dominated judgments made about these data sets. Experiments 3 and 4 further supported this interpretation. Experiment 3 showed an effect of Cell A frequency not predicted by any other model. Experiment 4 ruled out the alternative interpretation that the effect could be attributed to the manipulation of sample size.

Equation 2 was derived from the weighted averaging model for two causes proposed by White (2008). The four kinds of contingency information have relative weights that match those that have been found in several studies, but the model errs in assigning zero weight to Cell D. This discrepancy can be resolved by considering a further respect in which the $p \mathrm{CI}$ and $w p \mathrm{CI}$ models are oversimplifications: individual differences.

Under the present account, the activity of causal judgment reflects acquired understanding of the relevance of different kinds of contingency information, so that mean- 
ingful individual differences are likely to occur. Several studies have reported substantial individual differences in causal judgment (Anderson \& Sheu, 1995; Kao \& Wasserman, 1993; Levin et al., 1993; White, 2000a), and there are several reasons, within the context of the AFO model, why such individual differences might occur. The possibility most relevant to the issue of Cell $\mathrm{D}$ information is that there may be different conceptualizations of the use of each kind of information in strength estimation.

There are several ways in which strength can be estimated (Griffiths \& Tenenbaum, 2005; White, 2008). White (2008) proposed that strength estimation is based primarily on a comparison between Cell A and Cell B information, but this was not proposed as a normative procedure, only as being descriptive of a majority of individuals. Some individuals may recognize that information about what happens if the $\mathrm{CC}$ is absent has relevance to strength estimation. The evidence indicates that most of these individuals do not compute contingency; instead they weigh evidence, treating Cells $\mathrm{A}$ and $\mathrm{D}$ as confirmatory and Cells $\mathrm{B}$ and $\mathrm{C}$ as disconfirmatory, in the manner modeled by the $p \mathrm{CI}$ or $w p \mathrm{CI}$ rules. A smaller minority give equal weight to all four cells (Kao \& Wasserman, 1993). Such individuals could be comparing occurrence rates if the $\mathrm{CC}$ is present and if it is absent, but whether they conceptualize what they are doing as computing contingency or estimating strength is an open question. In any case, the presence of a minority of individuals who assess strength either from the proportion of confirmatory evidence or from the comparison of occurrence rates would be sufficient to raise the mean weight of Cell D to above zero. ${ }^{2}$

Individual differences of this and other kinds would also have the effect of reducing the differences between the weights given to the other three cells. The weights in Equation 2, although correctly reflecting the rank ordering of weights revealed by research, suggest greater differences in weights than tend to be observed. Cell B, for example, tends to carry more weight than Cell $\mathrm{C}$ but not by much (Kao \& Wasserman, 1993; Levin et al., 1993). This could be partly accounted for by the presence of individuals who weight all four kinds approximately equally for the purpose of strength estimation. It could also be the case that some individuals give more weight to accounting for occurrences than to estimating strength, and others give more to estimating strength than to accounting for occurrences. For the former, Cell C would carry more weight than Cell B, and for the latter, the opposite would be the case. Kao and Wasserman found that some individuals gave more weight to Cell $\mathrm{C}$ than to Cell $\mathrm{B}$, whereas the opposite was the case for others, which is consistent with this possibility.

Much remains to be learned about the meaning of individual differences in causal judgment. Given the evidence for them, however, individual differences of the kinds discussed here would tend to ameliorate otherwise considerable differences in weights between the four cells, bringing the AFO model more into line with research evidence. I would therefore propose that the weights shown in Equation 3 would make a better predictive model than those in Equation 2, while preserving the implications for cell weights of the principles of accounting for occurrences and estimating strength.

$$
J_{\mathrm{A}}=\frac{(.4 n \mathrm{~A})-(.3 n \mathrm{~B})-(.2 n \mathrm{C})+(.1 n \mathrm{D})}{(.4 n \mathrm{~A})+(.3 n \mathrm{~B})+(.2 n \mathrm{C})+(.1 n \mathrm{D})} .
$$

It needs to be emphasized that the weights in the model are not fixed and can vary if the variations do not violate the founding principles of the model. In particular, the weights do not necessarily hold for data sets with low proportions of CC-present information, which are considered in the next section. The weights also do not take account of effects of extrinsic factors such as the wording of the causal judgment question (White, 2003a).

\section{Extension of the Model to Data Sets With a Low Number of CC-Present Instances}

If little CC-present information is available, extreme judgments will not be made, because the small number of Cell A instances means that the CC cannot account for a large number of occurrences no matter what other information is available. In addition, strength estimation is based mainly on CC-present information, and a small amount of CC-present information is not subjectively adequate for an extreme estimate of strength. This has two consequences. One is that, if little CC-present information is available, only a limited range of the rating scale is considered to be available for judgment. People do not make an extreme judgment until they have a subjectively adequate sample, no matter what the proportion of Cell $\mathrm{A}$ to Cell C instances (or to Cell B instances, for strength estimation). The other consequence is that CC-present information carries more weight if there is little of it, because it is the kind of information that people want in order to resolve the aforementioned uncertainties in accounting for occurrences and estimating strength. They have a lot of CC-absent information, but they need more CC-present information to fulfill the aims of judgment. As CC-present information accumulates, so the weights gradually resolve to those given in Equation 3.

In the absence of research designed to investigate this, it is not easy to stipulate either the range of the rating scale that is considered to be available at different frequencies of CC-present information or the weights and how they change as judgment changes. Research on effects of sample size on causal judgment suggests that the subjectively adequate sample is small, probably no more than eight instances (Clément, Mercier, \& Pastò, 2002; Mercier \& Parr, 1996). Let us therefore assume that it is eight. If we further assume that the range of the scale considered to be available for judgment tends to increase as a linear monotonic function of CC-present frequency up to the maximum of eight, then, in effect, if there is just one CC-present instance, the maximum mean judgment observed should be 12.5 (on a scale from -100 to +100 ); if there are two CC-present instances, it should be 25; and so on. A similar limitation should apply in the negative direction.

How should the weights change? If there are no CCpresent instances, it seems likely that the weight given to 
CC-absent information should be close to 0 : How can one make any kind of judgment about a $\mathrm{CC}$ if there is no information about instances in which that $\mathrm{CC}$ is present? So let us assume that the weights for Cells $C$ and $D$ equal the respective weights in Equation 3 divided by the number by which the sample of CC-present instances falls short of the subjectively adequate frequency. Thus, if there are three CC-present instances, which is five short of the subjectively adequate frequency, then $w_{\mathrm{C}}=.2 / 5=.04$. This entails a corresponding increase in weights for Cells A and $\mathrm{B}$. If the total weight sums to 1 , then the weight available to $\mathrm{A}$ and $\mathrm{B}$ is $1-w_{\mathrm{C}}-w_{\mathrm{D}}$, and it would be divided between Cells A and B in such a way as to preserve the ratio of their weights (4:3), as shown in Equation 3. So if there is one Cell A instance and no Cell B instances, then $w_{\mathrm{C}}=.2 / 7=.029$ and $w_{\mathrm{D}}=.1 / 7=.014$. The collective weight available for CC-present information is therefore $1-.029-.014=.957$, which yields a weight of .547 for Cell A and .410 for Cell B.

These weights are then substituted for those in Equation 3 . The result of computing with the resultant equation is then multiplied by the assumed maximum mean judgment. Take the first data set in Experiment 3, in which the cell frequencies are $\mathrm{A}=1, \mathrm{~B}=0, \mathrm{C}=6, \mathrm{D}=2$. With the weights computed as shown above, the equation yields a value of .537 for this data set. The assumed maximum mean judgment is 12.5 , because there is only one CC-present instance, and $.537 \times 12.5=6.72$. This is the predicted mean judgment for this data set.

Using the model as just described, predictions were generated for Experiment 3 and are shown in the righthand column of that table, headed "Predicted." This shows a good fit between predictions and observed means. In particular, predicted values tend to increase as sample size increases, they tend to be higher for the data sets with the higher value of $\Delta P$, and the predicted rate of increase with sample size increase is greater at the higher than at the lower value of $\Delta P$. The observed mean for the data set with $\Delta P=+.75$ and sample size $=9$ is higher than the theoretical maximum of 12.5. One possible explanation for this is that the change in the maximum possible rating for a given Cell A frequency might follow a negatively accelerated exponential function rather than a linear monotonic function. Support for this comes from research on acquisition of causal judgment, in which negatively accelerated acquisition profiles have been found in most studies (Shanks, 1985, 1987; Shanks, Lopez, Darby, \& Dickinson, 1996; Wasserman et al., 1996; White, 2000a): The case of increasing numbers of CC-present instances is functionally similar to a learning situation, so a negatively accelerated function could well apply.

Predictions were also generated for Experiment 4 and are shown in the right-hand column of Table 4 . The table shows that the model predicts the one significant finding of Experiment 4, the main effect of $\Delta P$, because all four predicted values at $\Delta P=+.25$ are higher than all four at $\Delta P=-.25$. The predicted values for $\Delta P=-.25$ show a slight declining tendency, which can also be seen in the observed means, but this trend was not statistically significant. Predicted values at $\Delta P=+.75$ are all within less than one scale point of each other, consistent with the lack of significant effect found there.

This extension to the AFO model is an attempt to reconcile the need to give greater weight to CC-present information if little is available with the tendency to avoid extreme judgment if there are few CC-present instances. Clearly there are other assumptions on which a model of this general sort could be constructed, such as the assumption of a negatively accelerated change in the permitted range of judgment as the number of Cell A instances increases. But these are matters of fine-tuning; the main point is that the model does predict the main features of the results of Experiments 3 and 4.

\section{Normative Models of Causal Judgment}

The results disconfirm the basic prediction of the power $\mathrm{PC}$ theory that $\mathrm{CCs}$ with a positive contingency should be judged generative and those with a negative contingency should be judged preventive. The power PC theory may be somewhere near an extreme in its postulation of natural competence at causal judgment, but these results are problematic for any model that hypothesizes a reasonable degree of competence at contingency assessment in causal judgment, because the judgmental tendencies found here are unambiguously wrong by the standard of the objective contingency. A generative $\mathrm{CC}$ cannot decrease the likelihood of its outcome's occurring, nor can a preventer increase the likelihood of the outcome it prevents. Either people are not good at assessing contingencies, or they are not assessing contingencies at all. I have argued for a version of the latter: that selected kinds of contingency information are used as evidence with respect to specific aims of causal judgment and that this activity does not involve the assessment of contingency.

One possible counter to this would be that models such as the power PC theory recognize a distinction between competence and performance. Causal judgments could deviate from normative prescriptions if task demands impose a cognitive load on participants that exceeds their processing capacity. However, it is not likely that this could apply in the present case. In these experiments, the instance list procedure was used. In this, all instances are available for inspection at once, so there is no demand on memory capacity. Participants are able to scan the list of instances for as long as they wish. The instances, although randomly ordered, are laid out in a form convenient for the assessment of contingency. The presentation has a clear set of associations between presence and absence of the $\mathrm{CC}$ and occurrence or nonoccurrence of the outcome, as the example in the Appendix shows. Anybody whose natural means of making causal judgments is by assessing contingency should find this much less demanding than contingency assessment under the conditions of ordinary life, where relevant observations are scattered among multitudinous other events and distributed across a wide temporal range. Processing limitations might add a certain amount of noise to causal judgments, perhaps reducing the discriminability of different contingencies. But it is hard to see how an argument based on performance's not matching up to competence could account for significant differences in 
judgment that are contrary to the objective contingencies. This is surely a sign of a process that works in a different way from normative inference. So it is unlikely that arguments based on a distinction between competence and performance can save normative models in this case.

The results disconfirm the predictions of any model of causal judgment that is based on, or isomorphic with, $\Delta P$ values. An alternative to normative rule-based models such as the power PC theory is that causal judgments are derived from acquired strengths of associative bonds between CCs and outcomes. In essence, instances in which $\mathrm{CC}$ and outcome are both present tend to strengthen the associative bond between them and instances in which the CC is present but the outcome does not occur tend to weaken it. The associative learning model that has been most investigated in application to human causal judgment is the Rescorla-Wagner model (Allan, 1993; De Houwer \& Beckers, 2002; Rescorla \& Wagner, 1972; Tangen \& Allan, 2003). It has been shown that asymptotic values generated by the Rescorla-Wagner model are isomorphic with values of $\Delta P$ (Allan, 1993; Tangen \& Allan, 2003). The Rescorla-Wagner model therefore predicts higher causal judgments for positive than for negative contingencies. This prediction is disconfirmed by the present results. It is likely that the same would hold for any associative learning model. If associative bonds are strengthened by Cell A instances and weakened by Cell B instances, then CCs with positive contingencies should always have stronger associative bonds with their outcomes than would CCs with negative contingencies.

\section{Assessment of Other Models}

Strong cases have been made for two other models as accounts of human causal judgment from contingency information - namely, the dual-factor heuristic (Hattori \& Oaksford, 2007) and the causal support model (Griffiths \& Tenenbaum, 2005). It is therefore important to assess whether these models can account for the present results.

The dual-factor heuristic. Hattori and Oaksford (2007) argued that, under real-world judgmental conditions, it is often impossible to define what counts as a Cell D instance or to observe or count Cell D instances. They therefore proposed that causal judgments are made in accordance with a heuristic that approximates the output of normative rules for contingency assessment, but without using Cell D information. This is the dual-factor heuristic, operationalized as the $H$ rule (Hattori \& Oaksford, 2007). In this rule, $p(\mathrm{E} / \mathrm{C})$ (the probability of the outcome given that the $\mathrm{CC}$ is present) is multiplied by $p(\mathrm{C} / \mathrm{E})$ (the probability of the $\mathrm{CC}$ being present given that the outcome has occurred), and the square root of the result is taken. The two conditional probabilities operationalize the two goals of explanation (how well does the presence of the CC predict the occurrence of the outcome) and prediction (how well are occurrences of the outcome explained by the presence of the CC). Note that Cell A enters both conditional probabilities in this rule, Cells $\mathrm{B}$ and $\mathrm{C}$ enter one each, and Cell $\mathrm{D}$ enters none.

Because its minimum value is 0 , one problem with the $H$ rule is that there is no objective distinction between val- ues that indicate causes and values that indicate preventers. It is only possible to test predictions of ordinal differences. Even so, the model fails to predict the results of Experiment 1. For Group 1, data set $\{1,4,8,24\}, H=.15$, and for Group 1, data set $\{4,1,24,8\}, H=.34$. The dualfactor heuristic therefore predicts higher judgments for the latter data set than for the former. However, there was no significant difference between their means $[F(1,43)=$ $\left.0.61, M S_{\mathrm{e}}=2,231.54\right]$. For Group 2 , data set $\{0,2,8,24\}$, $H=0$, and for Group 2, data set $\{2,0,24,8\}, H=.29$, so the dual-factor heuristic predicts higher judgments in the latter data set than in the former. Again, there was no significant difference $\left[F(1,43)=0.26, M S_{\mathrm{e}}=1,718.17\right]$. The results therefore did not confirm the predictions of the dual-factor heuristic.

The predictions of the $H$ rule for Experiments 3 and 4 are shown in Tables 3 and 4, respectively. Table 3 shows that the $H$ rule predicts no effect of the sample size manipulation in Experiment 3. Within each contingency, values of $H$ are identical at all sample sizes. However, a significant effect was found. Table 4 shows that the $H$ rule predicts that causal judgments should tend to decrease as sample size increases in Experiment 4. However, no significant effect was found. These results are therefore disconfirmatory for the predictions of the $H$ rule.

Hattori and Oaksford (2007, Experiment 2) reported results showing that the $H$ rule tended to be better than the $p \mathrm{CI}$ rule as a predictor of causal judgments. The experimental stimuli comprised data sets for which $H=.2, .5$, and .8 , with three data sets at each value of $H$. Using the weights used in the present Experiment 1, computations of $w p C I$ confirm the tendencies for that rule reported by Hattori and Oaksford. If $H=.8, w p C I$ successfully predicts differences in judgment between the three data sets in line with their findings (Figure 2 in Hattori \& Oaksford, 2007). At that level, therefore, $w p C I$ was a better predictor than was the $H$ rule. There were comparatively large Cell A frequencies in those three data sets $(12,12$, and 9), so this result is consistent with the contention that the $w p \mathrm{CI}$ rule is a good predictor of causal judgments if there are adequate numbers of Cell A instances. At the other values of $H$, however-. 2 and .5 - the predictions of $w p \mathrm{CI}$ were not supported. Those are the results that are of most interest here. The CC in question does a better job of accounting for occurrences when $H=.5$ than when $H=.2$, partly because there are more Cell A instances when $H=.5(3,3$, and 1$)$ than when $H=.2(1,1$, and 1$)$ and partly because there are fewer Cell $\mathrm{C}$ instances when $H=.5(6,6$, and 3$)$ than when $H=.2(12,24$, and 19$)$. The AFO model therefore predicts higher judgments for the three data sets for which $H=.5$ than for the three for which $H=.2$. The results fit with these predictions. The AFO model therefore appears capable of accounting for both the results reported by Hattori and Oaksford and those reported here, whereas the $H$ rule fails to account for those reported here.

The dual-factor heuristic and the AFO model superficially resemble each other in that both postulate two distinguishable judgmental tasks, one calling on Cells A and $\mathrm{C}$ and one calling on Cells A and B. But, in fact, the 
tasks postulated in the two models are not that similar. There are two main differences:

1. The $H$ rule uses only the proportions minus the conditional probabilities. In the AFO model, there is a balance to be struck between proportions and frequencies: The predictions of $w p C I$ break down at low $p(\mathrm{C})$ because, if there are few Cell A instances, frequency information becomes more important and proportion information less so.

2. In the $H$ rule, the two conditional probabilities capture the tasks of prediction and explanation. Prediction is not problematic: $p(\mathrm{E} / \mathrm{C})$ is a straightforward measure of the extent to which the presence of the $\mathrm{CC}$ predicts the outcome. Explanation is problematic: $p(\mathrm{C} / \mathrm{E})$ is not a straightforward measure of the extent to which the outcome is explained by the $\mathrm{CC}$, because this depends on whether other possible causes are also present. In the AFO model, things are the other way around: Accounting for occurrences is not problematic, because the extent to which the $\mathrm{CC}$ is present when the outcome occurs is a straightforward measure of this - there is no implication of any concept of explanation. But estimating strength is problematic: $p(\mathrm{E} / \mathrm{C})$ is not a straightforward measure of strength, because it can be argued that the strength of a $\mathrm{CC}$ can be properly assessed only by comparing what happens if it is present with what happens if it is absent.

Thus, despite the similarity in the kinds of information used in causal judgment, the models are conceptually different and use the same kinds of information for different purposes. In the present case, the predictions generated by the two models diverge primarily because the $H$ rule uses only proportions and weights them equally. The AFO model stipulates different cell weights, but under this model, judges seek a balance between proportion information and frequency information; if there are few or no Cell A instances, the balance shifts toward frequency information.

The causal support model. Tables 1 and 2 show values of support, which are the predictions of the causal support model (Griffiths \& Tenenbaum, 2005). The model is briefly and informally summarized here; a full account can be found in Griffiths and Tenenbaum. Causal support is a Bayesian model of causal judgment. That is, the reasoning is based on Bayes's theorem, which is a formal model of the updating of prior beliefs in the light of evidence. The causal support model distinguishes two kinds of judgments: structure and strength. In the case in which one explicit $\mathrm{CC}$ is under consideration, the structure problem concerns distinguishing between two graphs that describe the possible structure of the system. In each graph, there are three nodes: one for the explicit $\mathrm{CC}$, one for the outcome, and one representing the set of other possible causes of the outcome. Both graphs have a link from the set of other possible causes to the outcome. In one graph, there is a link from the explicit causal candidate to the outcome; in the other, there is not. The structure problem involves deciding which of these graphs describes the structure of the system. The strength of a link cannot be assessed until the presence of the link in the graph has been ascertained. In the absence of strength assessment, this is accomplished in the causal support model by the in- tegration of evidential support for the link across all possible values of strength. Thus, contingency information is used in this integration process to ascertain which graph represents the structure of the system and is then used to assess the strength of the link between the $\mathrm{CC}$ in question and the outcome, if that link is judged to be there.

There are two support measures, a generative form that assesses the evidence for a generative causal link as opposed to no link and a preventive form that assesses the evidence for a preventive causal link as opposed to no link. Ideally, choice between these two should be made on the basis of whether participants were asked to assess the generative or preventive status of the $\mathrm{CC}$. In the present case, this cannot be done, because a single measure was used, on which participants could make either generative or preventive judgments. Instead, predictions of the model were generated by choices made on the basis of the objective contingency: The generative form was used for data sets with a positive contingency, and the preventive form was used for data sets with a negative contingency.

Values of causal support are unbounded, and the causal support model tends to predict large effects of sample size, which are not found (e.g., Lober \& Shanks, 2000; White, 2002). To ameliorate predicted sample size effects, the predicted values in Tables 1-3 were generated by using a power transformation of the form $j=$ $\operatorname{sign}\left(\right.$ support) $\cdot$ abs(support) ${ }^{k}$, as recommended by Griffiths and Tenenbaum (2005). I followed the procedure used by Perales and Shanks (2007), searching $k$ in intervals of 0.05 to find the maximum fit between support and judgments. Across the data sets used in Experiments 1 and 2 , the best fit was found at $k=.35$; those are the values reported herein.

For Experiment 1, Table 1 shows that causal support values in both groups were higher at the higher value of $p(\mathrm{C})$ than at the lower value of $p(\mathrm{C})$ but were not affected by the manipulation of $p(\mathrm{E})$. In both groups, the two means for the first two data sets in the table were predicted to be higher than the other two. As Table 1 shows, in both groups, they were in fact intermediate between the other two. Therefore, the causal support model fails to predict the results of this experiment. In Experiment 2, Table 2 shows that values of support at the high sample size matched the ordinal difference in the means, but values of support at the low sample size were identical, so that prediction of no difference was disconfirmed by the significant difference found. The causal support values also show a predicted interaction between $\Delta P$ and sample size, which was not found.

It could be argued that the question asked of participants concerned causal strength, not causal structure. According to the causal support model, the issue of strength arises only after the structure question has been decided. Thus, the model might not apply to cases in which participants are asked only about strength. It is yet to be established that people distinguish between structure and strength as defined under the causal support model. On the assumption that they do, if strength judgments depend on structure judgments, then participants making nonzero judgments of strength must ipso facto have decided that 
there was indeed a link between the $\mathrm{CC}$ and the outcome. According to the model, one cannot assess the strength of a link without having decided that it exists, so a nonzero assessment of strength implies that a judgment has been made that the link exists. In Experiment 1, taking the eight data sets in the order of presentation in Table 1, numbers of participants making judgments of zero were 10, 8, 0, 2, 15, 12, 1, and 3. In Experiment 2, taking the four data sets in the order of presentation in Table 2, numbers of participants making judgments of zero were $4,8,2$, and 3 , and no participant made a judgment of zero for more than two of the four data sets. All participants in Experiments 1 and 2 therefore judged, implicitly at least, that a link existed in at least one data set, and most of them made that judgment for all the data sets that they encountered.

The structure question in the causal support model asks people to choose either between a generative link and no link or between a preventive link and no link. The measure used in the present research asks participants to choose whether to identify the $\mathrm{CC}$ as generative or preventive, so it could be argued that this measure does not map onto the structure task. However, the center point of the rating scale (0) was anchored by the expression "has no effect" (see the Method section of Experiment 1), which enables participants to choose either between a generative link and no link or between a preventive link and no link. In either case, they could make a judgment of 0 if they chose the structure in which there was no link. So the rating scale does enable hypothetical causal support judges to make judgments that express both structure (i.e., distinguishing between either kind of link and no link) and strength. A judgment that there is no link can be expressed by a rating of 0 , a judgment of a generative link by some number between 1 and 100, and a judgment of a preventive link by some number between -1 and -100 .

For the present analysis, the generative support model was chosen if the objective contingency was positive, and the preventive support model was chosen if the objective contingency was negative. As a Bayesian model, causal support models how beliefs change in light of evidence. It could therefore be argued that, because participants were not asked to evaluate one model or the other directly, the choice of model should depend on the participants' prior beliefs. It is not clear that the participants would have any particular prior belief; even if they did, it would not be correlated with the objective contingency they encountered, because that contingency varied unpredictably from one data set to another as a result of the random ordering of data sets. It is likely that any prior belief would approximate to zero, which could be taken as justifying a decision to base the choice of support model on whether the contingency encountered was positive or negative. It could be argued that the model cannot be applied to a situation in which participants were not asked to judge on the basis of one model or the other - for example, judging whether there is a generative link between the $\mathrm{CC}$ and the outcome. On the other hand, Griffiths and Tenenbaum (2005) claimed that their model does apply to studies using strength measures similar to that used here. It is therefore likely that the results do indicate a failure of the causal support model.

\section{The Rationality Issue}

The results of these experiments could be taken as implying that human causal judgment is biased or imperfect. There is, however, a difference between rationality and conformity to normative principles. Judgmental tendencies of the kinds observed here could be rational without being normative. There are many possible definitions of rationality, and the nature of rationality is complex (McKenzie, 2003; Stanovich, 1999; Stanovich \& West, 2000). Without committing oneself to a strong definition of rationality, it is possible to argue that judgment is rational in a limited sense if it optimally fulfills the aims in respect of which it was made (Anderson, 1990). Thus, if the aim is to account for occurrences of an outcome, it is arguably rational to focus on occurrences and to assess the possible causes that are present when outcomes occur.

Hattori and Oaksford (2007) argued that the dual-factor heuristic is rational in the sense that it is an optimal approximation in an uncertain world. In particular, it is often difficult, if not impossible, to define what counts as Cell D information outside the laboratory, because the class of instances in which the $\mathrm{CC}$ was not present and the outcome did not occur is usually unbounded. The dual-factor heuristic neglects Cell D information, but Hattori and Oaksford showed that it still generates an acceptable approximation to the normative measure of covariation, the phi coefficient. Such a rule would indeed be a rational means of coping with the difficulties of gathering relevant information and assessing contingency if assessing contingency were the aim of judgment. But if the twin aims of judgment are accounting for occurrences and estimating strength, a rule of judgment is rational to the extent that it is an optimal means of meeting those aims. Thus, the judgmental tendency observed here could be construed as rational in the sense that it is suited to the purposes for which contingency information is used in causal judgment. Even so, it is counternormative in the sense that it violates the prescriptions of any model that would postulate a reasonable degree of competence at assessing contingency in causal judgment.

Behavior that is rational outside the laboratory can appear to be irrational inside it. Outside the laboratory, what counts as Cell D information may be hard to define, but in most cases the Cell D frequency would be very large-larger out of all proportion to frequencies in the other three cells. For example, people who have neither a given virus nor a given disease usually greatly outnumber those who fall into the other three categories. Difficulties of defining and counting Cell D instances motivate the use of a heuristic that does not require Cell D information, and that is part of the rationale for the $H$ rule (Hattori \& Oaksford, 2007). In the laboratory, Cell D information is usually both as clearly defined and as easily counted as information of the other three kinds. Experimental studies of causal judgment take care of this problem by preparing clearly defined sets of stimulus information. Causal judgments then appear counternormative, because the stimuli present conditions under which the heuristic breaks down and fails to approximate the normative standard.

If accounting for occurrences and estimating strength are the main aims of causal judgment, ways of accomplishing 
these aims are likely to be adapted to the conditions that prevail outside the laboratory. Thus, to estimate the strength of a CC may, normatively, require some comparison between what happens if the CC is present and if it is absent; that is, it should involve all four cells. But if there are problems with defining and counting Cell D information, the comparison is forgone, and strength is estimated just from CC-present information, without any kind of comparison. The use of Cell D information in laboratory studies may then reflect a recognition by some participants (probably a minority; Anderson \& Sheu, 1995; White, 2000a) that it is relevant if it is well defined and available. It is not necessarily irrational for other participants to neglect Cell D information in the laboratory; it is just a perpetuation of their usual practice in the conditions that prevail outside the laboratory. This much is common to the dual-factor heuristic account (Hattori \& Oaksford, 2007) and to the AFO model. Nevertheless, the present results indicate that the AFO model is better able than the $H$ rule to account for judgmental tendencies that occur in cases with few Cell A instances.

It could be argued that causal judgment must be rational on the grounds that humans are generally successful in dealing with the world, which includes using causal judgments to infer regularities, make predictions, and exert some kind of control over events. But it is not clear that humans really are all that successful. All manner of false beliefs about causes can be found, particularly in areas of vital practical importance, such as health and disease, and these false beliefs lead people to take actions that are dangerous or detrimental and to avoid taking other actions that would be more beneficial. The human race as a whole is inflicting damage on the world, destroying ecosystems and food webs and altering global climate, with potentially very serious consequences; errors of causal judgment may play a part in this (White, 2000b). If causal judgments are made in the service of practical concerns, individuals may err by not having clear and appropriate ideas about what their best practical interests really are, as well as by making causal judgments in ways that are only approximately suited to the practical concerns they think they have. Thus, although the kind of causal judgment and its conceptual foundation that is represented in the AFO model may function adequately in the service of practical concerns, it is likely that it subserves only a limited and error-prone kind of rationality.

\section{AUTHOR NOTE}

I am grateful to Tom Griffiths and Jose Perales for advice on the use of the power transformation with the causal support model, to Marc Buehner for assistance with computing power transformed values of causal support, and to Tom Beckers, Marc Buehner, and Joshua Tenenbaum for helpful comments on an earlier version of this article. Address correspondence to P. A. White, School of Psychology, Cardiff University, P.O. Box 901, Cardiff CF10 3AT, Wales (e-mail: whitepa@cardiff.ac.uk).

\section{REFERENCES}

Allan, L. G. (1993). Human contingency judgments: Rule based or associative? Psychological Bulletin, 114, 435-448.

ANDERSON, J. R. (1990). The adaptive character of thought. Hillsdale, NJ: Erlbaum.
Anderson, J. R., \& Sheu, C.-F. (1995). Causal inferences as perceptual judgments. Memory \& Cognition, 23, 510-524.

BUSEMEYER, J. R. (1991). Intuitive statistical estimation. In N. H. Anderson (Ed.), Contributions to information integration theory (Vol. 1, pp. 187-215). Hillsdale, NJ: Erlbaum.

Catena, A., Maldonado, A., \& Cándido, A. (1998). The effect of the frequency of judgment and the type of trials on covariation learning. Journal of Experimental Psychology: Human Perception \& Performance, 24, 481-495.

Cheng, P. W. (1997). From covariation to causation: A causal power theory. Psychological Review, 104, 367-405.

Clément, M., Mercier, P., \& Pastò, L. (2002). Sample size, confidence, and contingency judgement. Canadian Journal of Experimental Psychology, 56, 128-137.

De Houwer, J., \& Beckers, T. (2002). A review of recent developments in research and theories on human contingency learning. Quarterly Journal of Experimental Psychology, 55B, 289-310.

Griffiths, T. L., \& Tenenbaum, J. B. (2005). Structure and strength in causal induction. Cognitive Psychology, 51, 334-384.

HATTORI, M., \& OAKSFORD, M. (2007). Adaptive non-interventional heuristics for covariation detection in causal induction: Model comparison and rational analysis. Cognitive Science, 31, 765-814.

KaO, S.-F., \& Wasserman, E. A. (1993). Assessment of an information integration account of contingency judgment with examination of subjective cell importance and method of information presentation. Journal of Experimental Psychology: Learning, Memory, \& Cognition, 19, 1363-1386.

Levin, I. P., Wasserman, E. A., \& KaO, S.-F. (1993). Multiple methods for examining biased information use in contingency judgments. Organizational Behavior \& Human Decision Processes, 55, 228250.

LOBER, K., \& SHANKS, D. R. (2000). Is causal induction based on causal power? Critique of Cheng (1997). Psychological Review, 107, 195212.

MANDEL, D. R., \& Lehman, D. R. (1998). Integration of contingency information in judgments of cause, covariation, and probability. Journal of Experimental Psychology: General, 127, 269-285.

McKenZIE, C. R. M. (1994). The accuracy of intuitive judgment strategies: Covariation assessment and Bayesian inference. Cognitive Psychology, 26, 209-239.

McKenzie, C. R. M. (2003). Rational models as theories-not standards-of behavior. Trends in Cognitive Sciences, 7, 403-406.

Mercier, P., \& PARR, W. (1996). Inter-trial interval, stimulus duration and number of trials in contingency judgments. British Journal of Psychology, 87, 549-566.

Novick, L. R., \& Cheng, P. W. (2004). Assessing interactive causal influence. Psychological Review, 111, 455-485.

Perales, J. C., \& Shanks, D. R. (2007). Models of covariation-based causal judgment: A review and synthesis. Psychonomic Bulletin \& Review, 14, 577-596.

Rescorla, R. A., \& Wagner, A. R. (1972). A theory of Pavlovian conditioning: Variations in the effectiveness of reinforcement and nonreinforcement. In A. H. Black \& W. F. Prokasy (Eds.), Classical conditioning II: Current research and theory (pp. 64-99). New York: Appleton-Century-Crofts.

SHANKS, D. R. (1985). Continuous monitoring of human contingency judgment across trials. Memory \& Cognition, 13, 158-167.

SHANKs, D. R. (1987). Acquisition functions in contingency judgment. Learning \& Motivation, 18, 147-166.

Shanks, D. R., Lopez, F. J., DARby, R. J., \& Dickinson, A. (1996). Distinguishing associative and probabilistic contrast theories of human contingency judgment. In D. R. Shanks, K. J. Holyoak, \& D. L. Medin (Eds.), The psychology of learning and motivation: Vol. 34. Causal learning (pp. 265-311). San Diego: Academic Press.

Stanovich, K. E. (1999). Who is rational? Studies of individual differences in reasoning. Hillsdale, $\mathrm{NJ}$ : Erlbaum.

Stanovich, K. E., \& West, R. F. (2000). Individual differences in reasoning: Implications for the rationality debate? Behavioral \& Brain Sciences, 23, 645-726.

Tangen, J. M., \& Allan, L. G. (2003). The relative effect of cue interaction. Quarterly Journal of Experimental Psychology, 56B, 279300 . 
Wasserman, E. A., Dorner, W. W., \& KaO, S.-F. (1990). Contributions of specific cell information to judgments of interevent contingency. Journal of Experimental Psychology: Learning, Memory, \& Cognition, 16, 509-521.

Wasserman, E. A., Elek, S. M., Chatlosh, D. L., \& Baker, A. G. (1993). Rating causal relations: The role of probability in judgments of response-outcome contingency. Journal of Experimental Psychology: Learning, Memory, \& Cognition, 19, 174-188.

Wasserman, E. A., KaO, S.-F., Van Hamme, L. J., Katagiri, M., \& Young, M. E. (1996). Causation and association. In D. R. Shanks, K. J. Holyoak, \& D. L. Medin (Eds.), The psychology of learning and motivation: Vol. 34. Causal learning (pp. 207-264). San Diego: Academic Press.

White, P. A. (1989). A theory of causal processing. British Journal of Psychology, 80, 431-454.

White, P. A. (2000a). Causal judgment from contingency information: Relation between subjective reports and individual tendencies in judgment. Memory \& Cognition, 28, 415-426.

White, P. A. (2000b). Naive analysis of food web dynamics: A study of causal judgment about complex physical systems. Cognitive Science, 24, 605-650

White, P. A. (2002). Perceiving a strong causal relation in a weak contingency: Further investigation of the evidential evaluation model of causal judgment. Quarterly Journal of Experimental Psychology, 55A, 97-114.

White, P. A. (2003a). Effects of wording and stimulus format on the use of contingency information in causal judgment. Memory \& Cognition, 31, 231-242.

White, P. A. (2003b). Making causal judgments from the proportion of confirming instances: The $p$ CI rule. Journal of Experimental Psychology: Learning, Memory, \& Cognition, 29, 710-727.

WhITE, P. A. (2004). Causal judgment from contingency information: A systematic test of the $p$ CI rule. Memory \& Cognition, 32, 353-368.

White, P. A. (2008). Accounting for occurrences: A new view of the use of contingency information in causal judgment. Journal of Experimental Psychology: Learning, Memory, \& Cognition, 34, 204-218.

\section{NOTES}

1. This term is used to distinguish the definition of causal power in the power PC theory, as the probability with which a CC produces its outcome (Cheng, 1997; Novick \& Cheng, 2004), from the definition of causal power in the causal powers theory, as a specific capacity to produce a certain kind of outcome (White, 1989). The latter definition is not probabilistic.

2. It is reasonable to ask why individuals would adopt different procedures for assessing strength and why judging only from Cells A and B would be the most common option. There are two possible explanations for this.

In cases in which the identity of the cause is not in doubt, information about what happens if that cause is present is sufficient for estimating its strength. Take the example of someone perceiving a stone smashing a window: There is likely to be little if any doubt that the stone caused the window to be smashed. Therefore, one could assess the strength of the stone (i.e., its window-smashing capacity) by taking a sample of windows and seeing how many of them are smashed by that stone (ideally under suitably controlled conditions). Different stones could be assessed in the same way, and this would support an ordinal estimation of the strengths of different stones. There is no need for CC-absent information. The general procedure of estimating strength from CC-present instances would therefore be the most natural and simplest procedure to use. It takes an extra degree of sophistication to appreciate that a comparison with CC-absent information is better if the identity of the cause on any given occasion is uncertain and to understand how to use CC-absent information for that purpose. Under the present account, this is an acquired form of understanding, not part of our natural equipment for causal judgment.

In addition, as Hattori and Oaksford (2007) pointed out, it is often difficult, if not impossible, to sample CC-absent information in a manner appropriate for purposes of comparison. Cell $\mathrm{D}$ information tends to be difficult to define and sample, which entails that the rate of occurrence of the outcome if the cause is absent is often impossible to assess with any degree of accuracy. Under such conditions, estimating strength from CC-present information alone is a reasonable heuristic.

\begin{tabular}{|c|c|c|c|c|c|c|c|c|}
\hline \multicolumn{9}{|c|}{$\begin{array}{c}\text { APPENDIX } \\
\text { Example Data Set }\end{array}$} \\
\hline \multicolumn{9}{|c|}{ Species 13} \\
\hline Aquarium & $\begin{array}{c}\text { MS } \\
\text { Present? }\end{array}$ & $\begin{array}{l}\text { Green } \\
\text { Spots? }\end{array}$ & Aquarium & $\begin{array}{c}\text { MS } \\
\text { Present? }\end{array}$ & $\begin{array}{l}\text { Green } \\
\text { Spots? }\end{array}$ & Aquarium & $\begin{array}{c}\text { MS } \\
\text { Present? }\end{array}$ & $\begin{array}{l}\text { Green } \\
\text { Spots? }\end{array}$ \\
\hline 1 & No & No & 13 & Yes & No & 26 & Yes & Yes \\
\hline 2 & No & No & 14 & No & No & 27 & No & No \\
\hline 3 & No & No & 15 & No & No & 28 & No & Yes \\
\hline 4 & No & No & 16 & No & No & 29 & No & No \\
\hline 5 & No & No & 17 & No & Yes & 30 & No & Yes \\
\hline 6 & No & No & 18 & Yes & No & 31 & No & No \\
\hline 7 & No & No & 19 & No & Yes & 32 & Yes & No \\
\hline 8 & No & No & 21 & No & No & 33 & No & Yes \\
\hline 9 & No & Yes & 22 & No & No & 34 & No & No \\
\hline 10 & No & No & 23 & No & No & 35 & No & No \\
\hline 11 & Yes & No & 24 & No & Yes & 36 & No & No \\
\hline 12 & No & Yes & 25 & No & No & 37 & No & No \\
\hline
\end{tabular}

How does MS affect the occurrence of green spots in this species of parrotfish?

Note-The stimulus information is presented here in three sets of columns to save space. In the actual materials, there was just one set of columns covering one side of an A4 sheet of paper.

(Manuscript received May 23, 2008;

revision accepted for publication January 12, 2009.) 